\title{
Glucose transporter expression in eutopic endometrial tissue and ectopic endometriotic lesions
}

\author{
Brett McKinnon ${ }^{1,2}$, Dominic Bertschi', Carlos Wotzkow ${ }^{2}$, Nick A Bersinger ${ }^{1,2}$, \\ Jakob Evers ${ }^{1}$ and Michael D Mueller ${ }^{1,2}$ \\ ${ }^{1}$ Department of Obstetrics and Gynecology, Inselspital, Berne University Hospital, Effingerstrasse 102, \\ Berne $\mathrm{CH}-3010$, Switzerland \\ ${ }^{2}$ Department of Clinical Research, University of Bern, Murtenstrasse 35, Bern $\mathrm{CH}-3010$, Switzerland
}

Correspondence should be addressed to B McKinnon Email brett.mckinnon@ dkf.unibe.ch

\begin{abstract}
Endometriosis is an extremely prevalent disorder characterized by the growth of endometrial tissue at ectopic locations. Glycolysis is an energy-producing mechanism that occurs in almost all cells and requires an adequate uptake of glucose mediated by glucose transporter (GLUT) proteins. At present, however, very little is known about their expression in either the endometrium or the endometriotic lesions. The objective of this study was to examine the expression of SLC2A genes in the endometrium of women with and without endometriosis and in the matching ectopic tissue, and to confirm the presence of the GLUT proteins in ectopic lesions. There was a significantly higher expression of SLC2A3 and a significantly lower expression of SLC2A4 in women with endometriosis compared with those without. In women with endometriosis, the ectopic expression of SLC2A3, SLC2A4 and SLC2A5 was significantly higher than that observed in the matching eutopic tissue. GLUT1 protein expression was present in both epithelial and stromal cells and GLUT3 was confined to CD45-positive leukocytes. GLUT4 expression was strong in both ectopic epithelial and stromal cells and localized to the cellular membrane in epithelial cells. These results show that GLUT expression is altered between eutopic and ectopic tissue and between women with and without endometriosis, and that GLUT4 may represent a significant entry route for glucose into the endometriotic epithelial cells. The inducible nature of GLUT4 and its limited cellular expression may make GLUT4 an attractive target for non-hormone-based treatments of endometriosis.
\end{abstract}
Key Words
- endometriosis
- GLUT4
- endometrium
- glucose
- SLC2A

Journal of Molecular Endocrinology (2014) 52, 169-179

\section{Introduction}

Endometriosis is an extremely prevalent estrogendependent gynecological disorder affecting at least $10 \%$ of reproductive-aged women worldwide (Eskenazi \& Warner 1997). It is characterized by the growth of endometrial tissue outside the uterine cavity and can result in severe pelvic pain (Evans et al. 2007) and subfertility (D'Hooghe et al. 2003). Currently, the most widely accepted theory of endometriosis etiology purports that retrograde menstruation allows the implantation of viable endometrial cells at ectopic sites (Sampson 1927, Halme et al. 1984). Ectopic 
lesions then secrete chemotactic molecules that recruit immune cells, further stimulating the continued growth of the lesion.

Glycolysis is an energy-producing mechanism that requires an adequate supply of intracellular glucose uptake mediated by the glucose transporter (GLUT) proteins encoded by the $S L C 2 A$ gene family. These proteins transport glucose into the cell via a facilitated diffusion down a concentration gradient (Scheepers et al. 2004). Cloning of the first gene to encode a GLUT protein occurred in 1985 (Mueckler et al. 1985), after which a further 13 related members of the SLC2A gene family were subsequently identified in human (Uldry \& Thorens 2004).

Little information is available on GLUT expression in both endometrium and endometriotic lesions. GLUT1 is a ubiquitously expressed protein, responsible for the basal glucose uptake and is the most prominent GLUT in the endometrium (Frolova \& Moley 2011b). The highaffinity GLUT3 transporter has also been reported in eutopic endometrial tissue, although its expression is confined to CD45-positive leukocytes (von Wolff et al. 2003, Korgun et al. 2005). The insulin-sensitive GLUT4 has also been identified in endometrial tissue, but with contradictory results. No RNA expression was detected in endometrial biopsies (von Wolff et al. 2003) or in isolated endometrial stromal cells (Frolova \& Moley 2011a), although it has been detected in epithelial endometrial cells (Mioni et al. 2004, Mozzanega et al. 2004). The mRNA expression of the GLUT6, GLUT8, GLUT10 and GLUT12 genes has also been identified in a single study (Frolova \& Moley 2011b), although their role in the endometrium has not been examined.

The objective of this study was therefore to examine both the eutopic endometrial tissue of women with and without endometriosis, as well as matching ectopic endometriotic lesions to determine the expression of the $S L C 2 A$ genes and their cellular localization.

\section{Subjects and methods}

\section{Sample collection and patient data}

Women with idiopathic infertility or pelvic pain were recruited for the study, and prior to laparoscopic surgery, informed consent, patient details and medication history were collected. All laparoscopies were performed in the proliferative phase of the menstrual cycle, and during surgery any endometriotic lesions identified were removed and their location noted. In a subset of patients, endometrial biopsies were also collected using a soft curette (Pipelle-de-Cornier, Laboratoire CCD, Paris, France) and were split into two groups based on the presence or absence of histologically-confirmed endometriosis. No endometrial biopsies were included if the patients reported hormonal medication use in the 3 months prior to surgery. All tissue collected was stored in RNAlater at $-80^{\circ} \mathrm{C}$ until further use.

In cases were an ectopic endometrial lesion was identified, irrespective of whether a matching biopsy was collected, all ectopic tissue removed during surgery and collected and stored in RNAlater (Invitrogen Life Technologies) at $-80^{\circ} \mathrm{C}$ for subsequent analysis, as was the peritoneal fluid. For subsequent analysis of ectopic tissue, all samples collected were first split into groups based on hormonal treatment including no hormonal medication, estrogen- or progesterone-based hormonal contraceptives (HCs), or gonadotropin-releasing hormone analogs (GnRHa). In addition, within the no hormonal treatment cohort, an additional three groups based on lesion location were established (peritoneal, ovarian and rectovaginal septum (RVS)). As only two lesions with no hormonal treatment were available from the RVS group, this region was excluded from the statistical analysis.

The presence of endometriosis was confirmed by histological examination, and the menstrual stage of women not using hormonal medication was confirmed by measuring progesterone in the peritoneal fluid with a RIA (Coata-count, DPC; Buhlmann Laboratories, Allschwil, Switzerland) (Supplementary Figure 1, see section on supplementary data given at the end of this article). The cutoff value was $27 \mathrm{nmol} / \mathrm{l}$. Institutional Review Board approval was obtained from the ethical committee prior to the commencement of the study.

\section{Analysis of SLC2A gene expression in endometrial and endometriotic tissue}

For both the eutopic endometrial biopsies and ectopic lesions, $\sim 30 \mathrm{mg}$ tissue was excised and homogenized in the FastPrep 120 tissue homogenizer $(30 \mathrm{~s}$ at $4.0 \mathrm{~m} / \mathrm{s}$ ) in cell lysis buffer (Qiagen). The remaining RNA isolation was performed with the RNAeasy Mini Kit (Qiagen) and TurboDNase (Ambion, Life Technologies, Zug, Switzerland) for genomic DNase digestion. One microgram of total RNA was reverse transcribed in a $25 \mu \mathrm{l}$ reaction with the Moloney murine leukemia virus reverse transcriptase (Promega) and random primers. The resulting cDNA was diluted 1:20 and the absence of genomic DNA confirmed with a reverse transcriptase-negative control.

Published by Bioscientifica Ltd. 
Table 1 Primer sequences used for real-time PCR

\begin{tabular}{|c|c|c|}
\hline Gene & GenBank no. & Forward \\
\hline GAPDH & NM_002046 & TGCACCACCAACTGCTTAGC \\
\hline$A C T B$ & NM_001101 & CTGGAACGGTGAAGGTGACA \\
\hline YWHAZ & NM_003406 & ACTTTTGGTACATTGTGGCTTCAA \\
\hline$R P L 13 A$ & NM_012423 & CCTGGAGGAGAAGAGGAAAGAGA \\
\hline$S L C 2 A 1$ & NM_006516 & AAGGTGATCGAGGAGTTCTACA \\
\hline$S L C 2 A 2$ & NM_000340 & TTGCTGGAAGAAGCATATCAGG \\
\hline$S L C 2 A 3$ & NM_006931 & GTTCCCCTCACTGGATGAAA \\
\hline SLC2A4 & NM_001042.2 & CGTCGGGCTTCCAACAGATA \\
\hline$S L C 2 A 5$ & NM_003039 & CAAGAAAGCCCTACAGACGC \\
\hline$S L C 2 A 7$ & NM_207420 & TGTGCAGGCATCTCCTACAG \\
\hline$S L C 2 A 8$ & NM_014580.3 & GTCCTCACCAACTGGCTCAT \\
\hline
\end{tabular}

\begin{tabular}{l} 
Reverse \\
\hline GGCATGGACTGTGGTCATGAG \\
AAGGGACTTCCTGTAACAATGCA \\
CGCCAGGACAAACCAGTAT \\
TTGAGGACCTCTGTGTATTTGTCAA \\
ATGCCCCCAACAGAAAAGATG \\
TGACTAATAAGAATGCCCGTGAC \\
TATTTGGATGGCTCTCCCAC \\
CGCAGAGAACACAGCAAGGA \\
AACAGCTTCAGCACGGAGAT \\
CGAAAACCTCGGTCATTGTT \\
CAAGCCAGAAGGCTCCATAG
\end{tabular}

\begin{tabular}{c} 
Product size (bp) \\
\hline 86 \\
96 \\
92 \\
125 \\
119 \\
148 \\
95 \\
89 \\
117 \\
97 \\
91
\end{tabular}

The quantitative real-time qPCR was performed with the Rotor-gene SYBR Green PCR Kit (Qiagen) in a Rotor-gene RG 2000 (Corbett Research, Sydney, NSW, Australia) under the following conditions: $95^{\circ} \mathrm{C}$ for $5 \mathrm{~min}$, followed by 40 cycles of $95^{\circ} \mathrm{C}$ for $5 \mathrm{~s}$ and $60^{\circ} \mathrm{C}$ for $10 \mathrm{~s}$. Primers were purchased from Microsynth (Balgach, Switzerland), and the sequences are defined in Table 1 . Specificity of amplification was confirmed by melt curve analysis.

The four most stable reference genes among all samples were selected via the geNORM Software program (Zwijnaarde, Ghent, Belgium) and the geometric mean of all four reference genes was used to normalize the expression of the genes of interest (Vandesompele et al. 2002). The reaction efficiency of each assay was determined via linear regression (Ruijter et al. 2009) and the relative mRNA expression calculated with the qBASEplus Software (Biogazelle, Zwijnaarde, Belgium).

The comparison between SLC2A gene expression in eutopic endometrium from women with and without endometriosis and the comparison between eutopic expression and ectopic expression in matching samples from women with endometriosis were both performed using the non-parametric Mann-Whitney $U$ test, as was the mRNA comparison between peritoneal and ovarian lesions. The comparison between hormonal treatments was performed with a non-parametric one-way ANOVA test with a post-hoc Dunn's multiple comparison tests for each group.

\section{Immunodetection in endometriotic lesions and surrounding tissue}

After RNA had been harvested from the ectopic tissue, any remaining parts of the samples were fixed in formalin and embedded into paraffin (FFPE) blocks. Previous studies have confirmed the effectiveness of using tissue stored in RNAlater for immunohistochemistry (Florell et al. 2001). For imaging studies, serial sections of $4 \mu \mathrm{M}$ thickness were cut from each FFPE block and mounted onto glass slides (SuperFrost, Braunschweig, Germany). Prior to analysis, the sections were dewaxed in xylene and rehydrated through a series of ethanol. Epitope retrieval for all antibodies was performed via heating slides in $10 \mathrm{mM}$ citrate buffer at pH 5.5 for $5 \mathrm{~min}$ in a $450 \mathrm{~W}$ microwave oven. Endogenous peroxidase activity was blocked by treating sections with $3 \%$ hydrogen peroxide $\left(\mathrm{H}_{2} \mathrm{O}_{2}\right)$ for $10 \mathrm{~min}$ and non-specific binding blocked by incubation with 3\% BSA in Tris-buffered saline (TBS; Tris $100 \mathrm{mM}$, $\mathrm{NaCl} 0.15 \mathrm{M}$; pH 7.4) for $30 \mathrm{~min}$.

Primary antibody incubations were performed overnight in TBS containing 3\% BSA with the following dilutions: 1:50 for rabbit anti-human GLUT1 and rabbit anti-human GLUT4, 1:25 for rabbit anti-human GLUT3, and 1:75 for rabbit anti-human CD45 (all from Abcam, Cambridge, UK). After incubation, slides were washed with TBS containing $0.1 \%$ Tween 20 (TBST) and subsequently incubated with affinity purified, biotin-conjugated goat anti-rabbit IgG (Dako, Glostrup, Denmark) for 90 min at room temperature. After washing, the sections were incubated with avidin-biotin-HRP complex (Vectastain ABC Kit, Vector Laboratories, Burlingame, CA, USA) for $45 \mathrm{~min}$. Detection of bound antibody was via 1-5 min incubation at room temperature with 3,3'-diaminobenzidine substrate. Slides were counterstained with hematoxylin and mounted in Aquatex (Merck). Negative controls were performed in the absence of primary antibody. Immunostained slides were photographed with a Nikon Eclipse E800 microscope (Nikon, Japan) at a magnification of $20 \times$ and $60 \times$, and the endometriotic lesions, large nerve fiber trunks and blood vessels were identified via the hematoxylin staining. Individual leukocytes were identified via CD45 reactivity.

For immunofluorescence, the sample was prepared exactly as described for the immunohistochemistry and co-staining was performed by incubating the sections

Published by Bioscientifica Ltd. 
with both the rabbit anti-human GLUT4 (1:25) and the goat anti-human cytokeratin 19 (CK19; 1:500 dilution, Santa Cruz Biotechnology) antibody in 3\% BSA solution of TBS. Secondary antibodies were anti-goat IgG Cy5 (Millipore, Zug, Switzerland) and anti-rabbit IgG Alexa-Fluor 488 (Invitrogen). Nuclei were stained with $4^{\prime} 6$-diamidino2-phenylindole (DAPI) and slides mounted with Prolong Gold Antifade (Invitrogen). Images were photographed with the Zeiss LSM 710 Confocal Microscope. Negative controls were performed in the absence of the primary antibodies.

\section{Results}

\section{Sample collection}

Endometrial biopsies were collected from 13 women who were confirmed to have no visible endometriotic lesions and 15 women with endometriosis. There was no significant difference in either the age or the BMI between the two groups (Table 2). We also collected a total of 18 ectopic lesions from the 15 endometriotic women, as multiple ectopic samples were collected from two women. One women had three lesions, two of which were peritoneal and one in the RVS; whereas the second women had two lesions, one each from the peritoneal cavity and the ovary.

Additional ectopic tissue without matching eutopic biopsy was collected; 48 samples in total. Of these samples, 24 were from women with no hormonal treatment and 12 of these lesions were located in the peritoneal region, ten on the ovaries, and only two in the RVS. To determine the effect of hormonal treatment on SLC2A gene expression, a further 14 samples were available from women taking estrogen- or progesterone-based $\mathrm{HC}$ and ten women using GnRHa.

For the immunoanalysis of ectopic lesions, sufficient remaining tissue was available from 19 patients. These samples included eight from women who were not using hormonal treatments, six using $\mathrm{HC}$, and five using GnRHa. The tissue collected for this study is listed in Table 2.

\section{SLC2A gene expression in eutopic and ectopic endometrial tissue}

There was a significantly increased expression of SLC2A3 in the endometrium of women with endometriosis compared with those without. By contrast, there was a significantly decreased expression of SLC2A4 in the endometrial tissue of women with endometriosis compared with women without endometriosis (Fig. 1A). No significant difference was observed between the two groups for any of the other genes examined. No expression of $S L C 2 A 2$ was observed in either group.

In women with endometriosis, there was a significant increase in SLC2A3, SLC2A4 and SLC2A5 observed in the ectopic lesions compared with their matching eutopic endometrium (Fig. 1B). No significant variation was observed in SLC2A1,SLC2A7 and SLC2A8. No expression was observed for $S L C 2 A 2$ in any of the ectopic or eutopic samples.

\section{SLC2A gene expression in endometriotic tissue from different locations and under hormonal treatments}

The comparison of mRNA expression of the SLC2A family in different regions was only performed in samples

Table 2 Clinical data and sample parameters

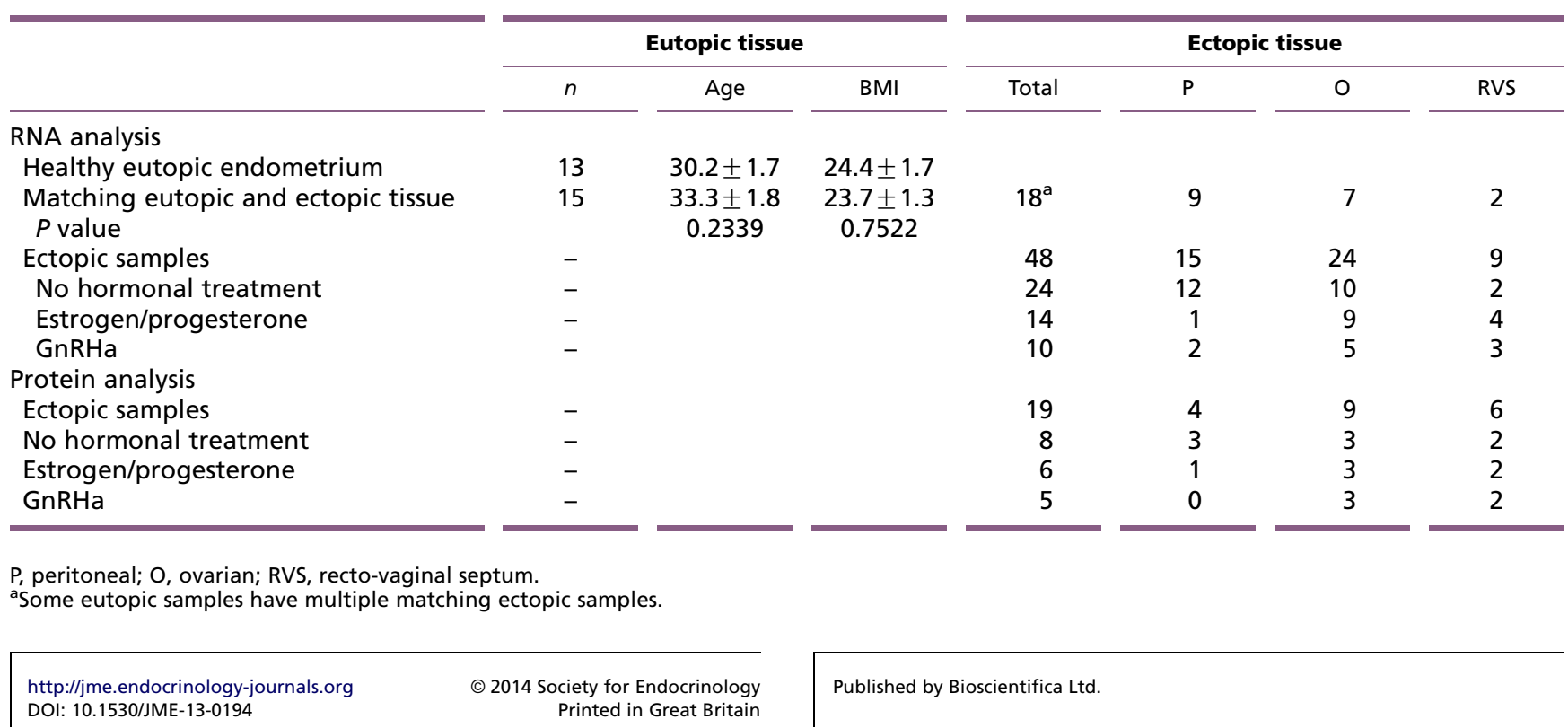



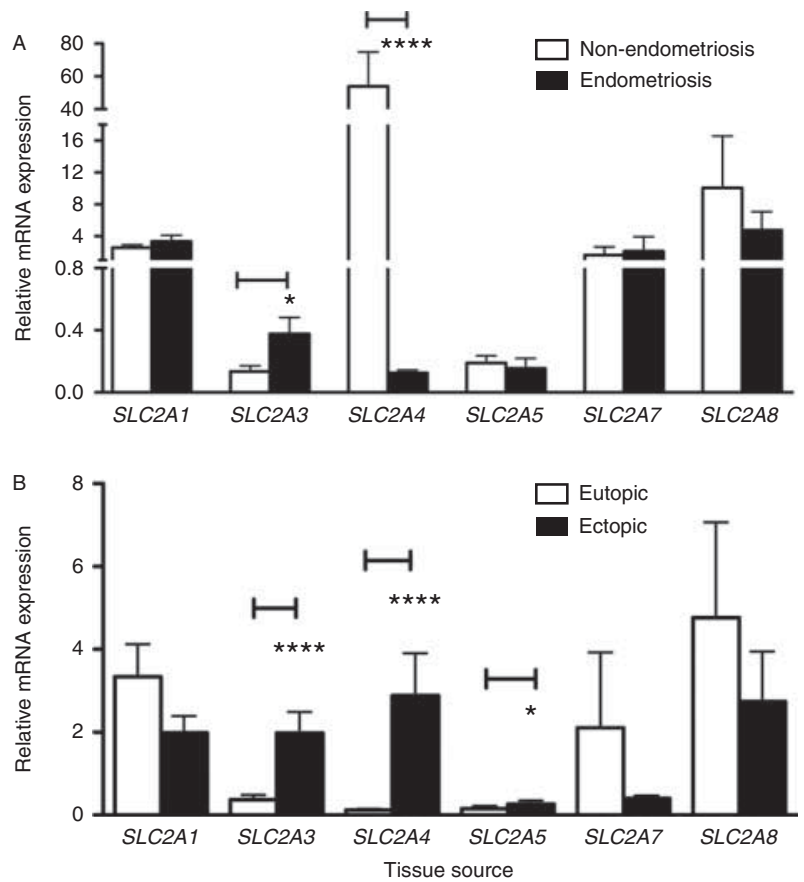

Figure 1

SLC2A gene expression in the eutopic and ectopic endometrial tissue of women with and without endometriosis. (A) A significantly higher expression of $S L C 2 A 3$ was observed in women with endometriosis $(0.38 \pm 0.11, n=15)$ compared with those without endometriosis $(0.13 \pm 0.04, n=13, P=0.0448)$. A significantly lower expression of SLC2A4 was observed in women with endometriosis $(0.13 \pm 0.02, n=15)$ compared with those without endometriosis $(53.87 \pm 21.07, n=13, P<0.0001)$. No significant difference for SLC2A1, SLC2A5, SLC2A7 or SLC2A8 was observed between women with or without endometriosis. No expression in either group was observed in $S L C 2 A 2$. (B) The eutopic expression of $\operatorname{SLC} 2 A 3$ ( $\mathrm{Eu}=0.38 \pm 0.11, n=15$ vs $\mathrm{EC}=1.98 \pm 0.51, n=18 ; P<0.0001)$, $S L C 2 A 4$ (Eu $=0.13 \pm 0.02, n=15$ vs $\mathrm{EC}=2.88 \pm 1.03, n=18 ; P<0.0001)$, and $S L C 2 A 5(\mathrm{Eu}=0.15 \pm 0.07, n=15$ vs $\mathrm{EC}=0.27 \pm 0.10, n=18 ; P=0.0464)$ was significantly lower than the paired ectopic expression. No significant change was observed in $S L C 2 A 1, S L C 2 A 7$ and $S L C 2 A 8$. All values are expressed as mean \pm s.E.M. ${ }^{*} P<0.05$ and $* * * * P<0.0001$.

from women who had not taken hormonal medication. Results show a significantly higher expression of both SLC2A1 and SLC2A8 in the peritoneal lesions compared with ovarian lesions (Fig. 2A). No significant difference was observed between any of the other genes examined.

An analysis on the effect of hormonal treatment on $S L C 2 A$ gene expression found no significant variation between any of the genes examined (Fig. 2B).

\section{GLUT1, GLUT3 and GLUT4 protein expression and their localization in endometriotic lesions and surrounding tissue}

In the endometriotic lesions, there was strong immunoreactivity of GLUT1 in both the epithelial and the stromal cells (Fig. 3A). In the stromal cells, immunoreactivity was predominantly nuclear, whereas epithelial cells showed both nuclear and membranous localization (Fig. 3B). GLUT3 showed only sparse immunoreactivity in scattered cells throughout the endometriotic lesions (Fig. 3C and D). The GLUT3 immunoreactivty coincided with the immunoreactivity observed for CD45 (Fig. 3E and F, black arrows). GLUT4 showed strong immunoreactivity in both the epithelial and the stromal cells of the endometriotic lesions (Fig. 3G). In the stromal cells this immunoreactivity appeared predominantly nuclear whereas the epithelial immunoreactivity was both nuclear, and membranous (Fig. 3H). No background signal was observed in the negative controls (Fig. 3I and J).

As dissected, endometriotic lesions will include structures other than endometrial tissue. Non-endometrial tissue, including blood vessels (Fig. 4) and large nerve fiber trunks (Fig. 5) were also examined in these samples. Strong GLUT1 immunoreactivity was observed in the endothelial cells lining the blood vessels surrounding the endometriotic lesions (Fig. 4A). This immunoreactivity appeared to be predominantly nuclear, with some membranous localization (Fig. 4B). GLUT3 showed very limited immunoreactivity in the cells surrounding the blood vessels (Fig. 4C and D). GLUT4 immunoreactivity was
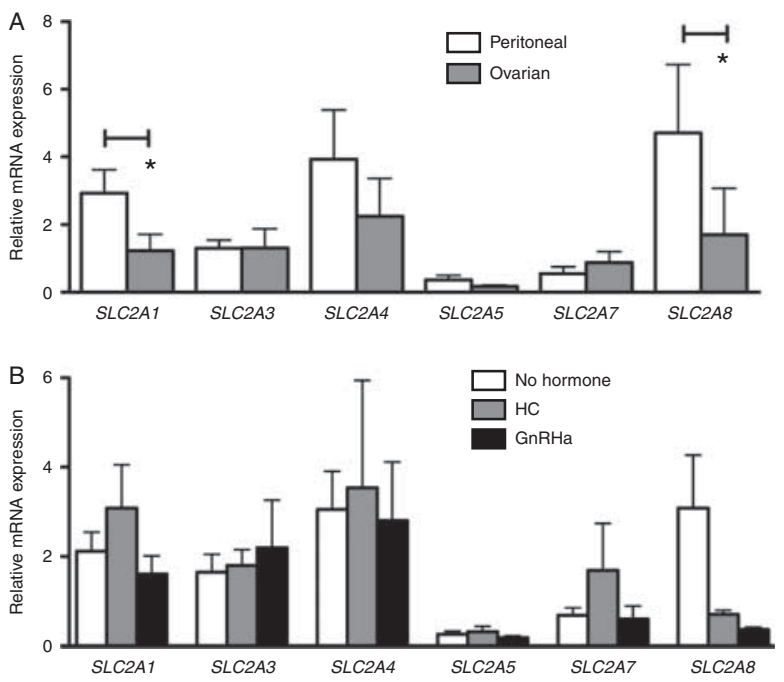

Figure 2

SLC2A gene expression in endometriotic lesions from different locations and under hormonal treatment. (A) A significantly stronger expression of $S L C 2 A 1$ (peritonal $=2.93 \pm 0.69, n=12$ vs ovarian $=1.23 \pm 0.49, n=10$; $P<0.011$ ) and SLC2A8 (peritoneal $=4.71 \pm 2.03, n=12$ vs ovarian $=1.71 \pm$ $1.37, n=10 ; P<0.019$ ) was observed in peritoneal lesions compared with ovarian lesions. No significant variation was observed in $S L C 2 A 3, S L C 2 A 4$, SLC2A5 and SLC2A7. (B) A comparison of SLC2A gene expression in the ectopic tissue of women using hormonal treatment found no significant variation in all genes examined. All values represent mean \pm s.E.M. ${ }^{*} P<0.05$.

Published by Bioscientifica Ltd. 

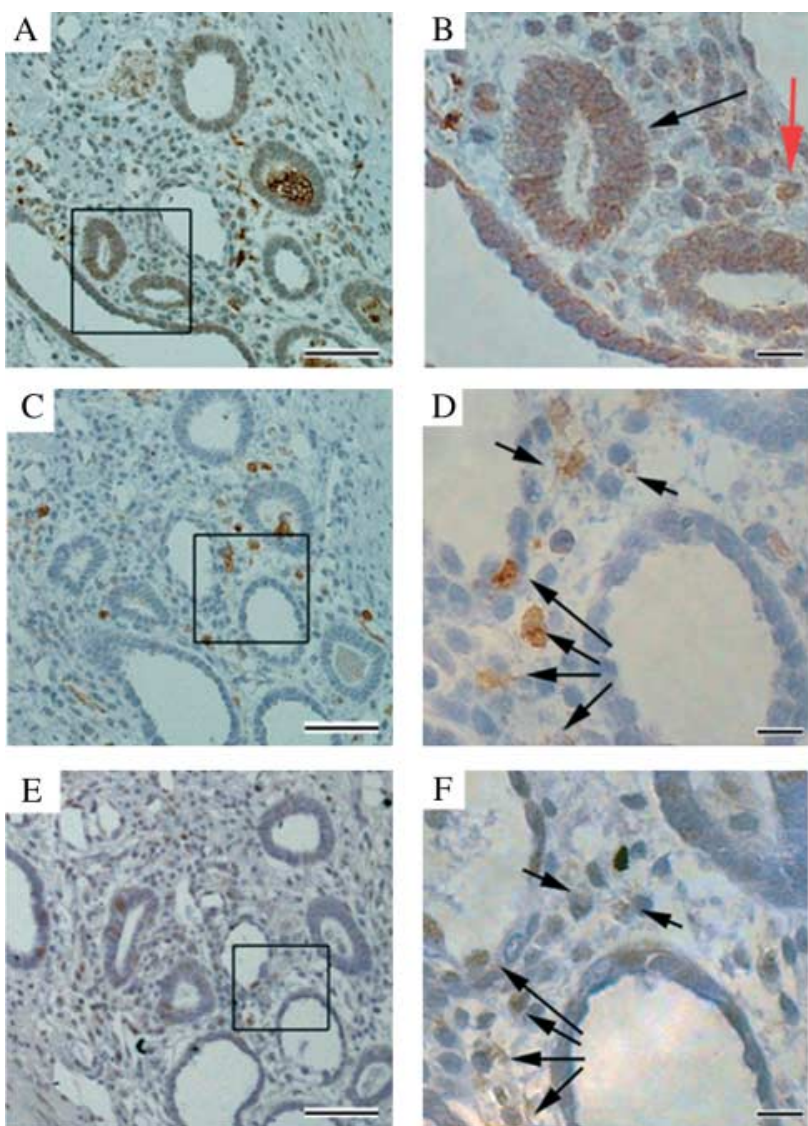
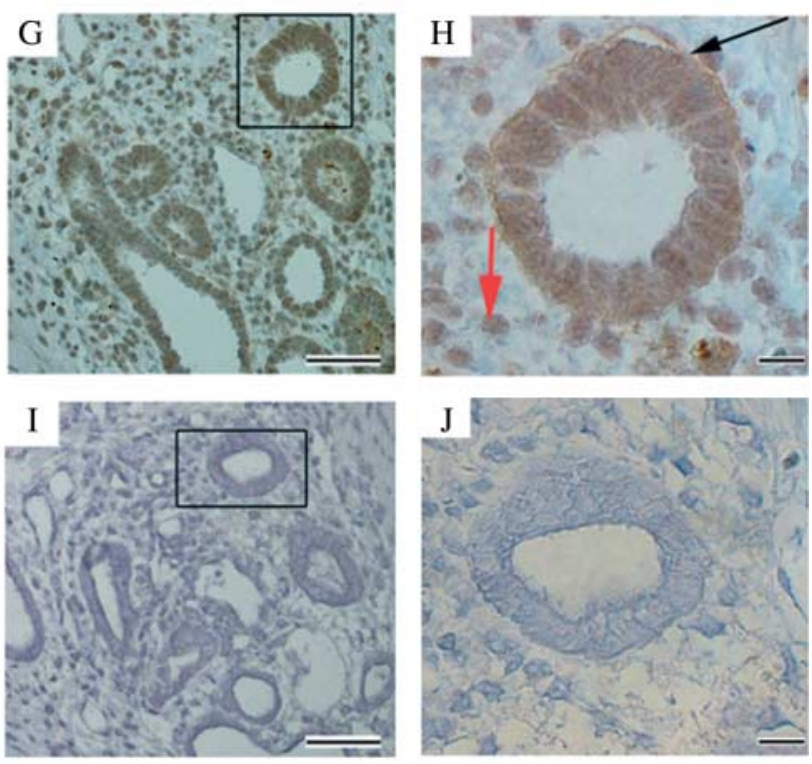

Figure 3

GLUT protein localization in endometriotic lesions. (A) GLUT1 immunoreactivity was detected in endometriotic lesions. (B) A higher magnification showed that moderate GLUT1 expression was present in both epithelial (black arrow) and stromal cells (red arrow). (C) Sparse GLUT3 immunoreactivity was observed in endometriotic lesions. (D) A higher magnification identified cells with GLUT3 immunoreactivity (black arrows). (E) CD45 immunoreactivity was observed within endometriotic lesions. (F) A higher magnification identified immunoreactivity of CD45 in cells

also observed in the endothelial cells lining the blood vessels (Fig. 4E and F). The negative control showed no background signal in the endothelial cells (Fig. 4G and H).

The large nerve fiber trunks showed a positive immunoreactivity to the GLUT1 antibody (Fig. 5A), which appeared to be predominantly nuclear, although with some immunoreactivity also in the cytosol and the membrane (Fig. 5B). GLUT3 showed some weak to moderate immunoreactivity in these large nerve fiber trunks (Fig. 5C), which was predominantly nuclear (Fig. 5D). GLUT4 immunoreactivity was also moderate to strong in the large nerve fiber trunks (Fig. 5E), although this immunoreactivity appeared to be almost exclusively nuclear (Fig. 5F). Negative controls showed no background signal (Fig. 5G and $\mathrm{H}$ ). similar to that of GLUT3 (black arrows). (G) Strong GLUT4 immunoreactivity was observed in endometriotic lesions. $(\mathrm{H})$ A higher magnification shows immunoreactivity is predominantly nuclear in the stromal cells (red arrow) and both nuclear and membranous in the epithelial cells (black arrow). (I and J) Negative control showed limited background staining in either epithelial or stromal cells. (A, C, E, G and I) Scale bars represent $50 \mu \mathrm{M}$ and the areas outlined in black represent the region of higher magnification. (B, D, F, H and J) Scale bars represent $10 \mu \mathrm{M}$.

\section{Localization of GLUT4 transporter protein to the membrane of endometriotic epithelial cells}

The nuclei of all cells in both the positive (Fig. 6A) and the negative control (Fig. 6B) were identified with DAPI. Immunofluorescence of GLUT4 (Fig. 6C), above the background and autofluorescence observed in the negative control (Fig. 6D), was identified in the epithelial cell membranes. Immunofluorescence of CK19 (Fig. 6E), above the background and autofluorescence observed in the negative control (Fig. 6F), was also observed in the epithelial cell membranes. An overlay of the images showed a significant co-localization (arrow) of the CK19 (red staining) and the GLUT4 (green staining) immunofluorescence in the epithelial membranes (Fig. 6G).

Published by Bioscientifica Ltd. 

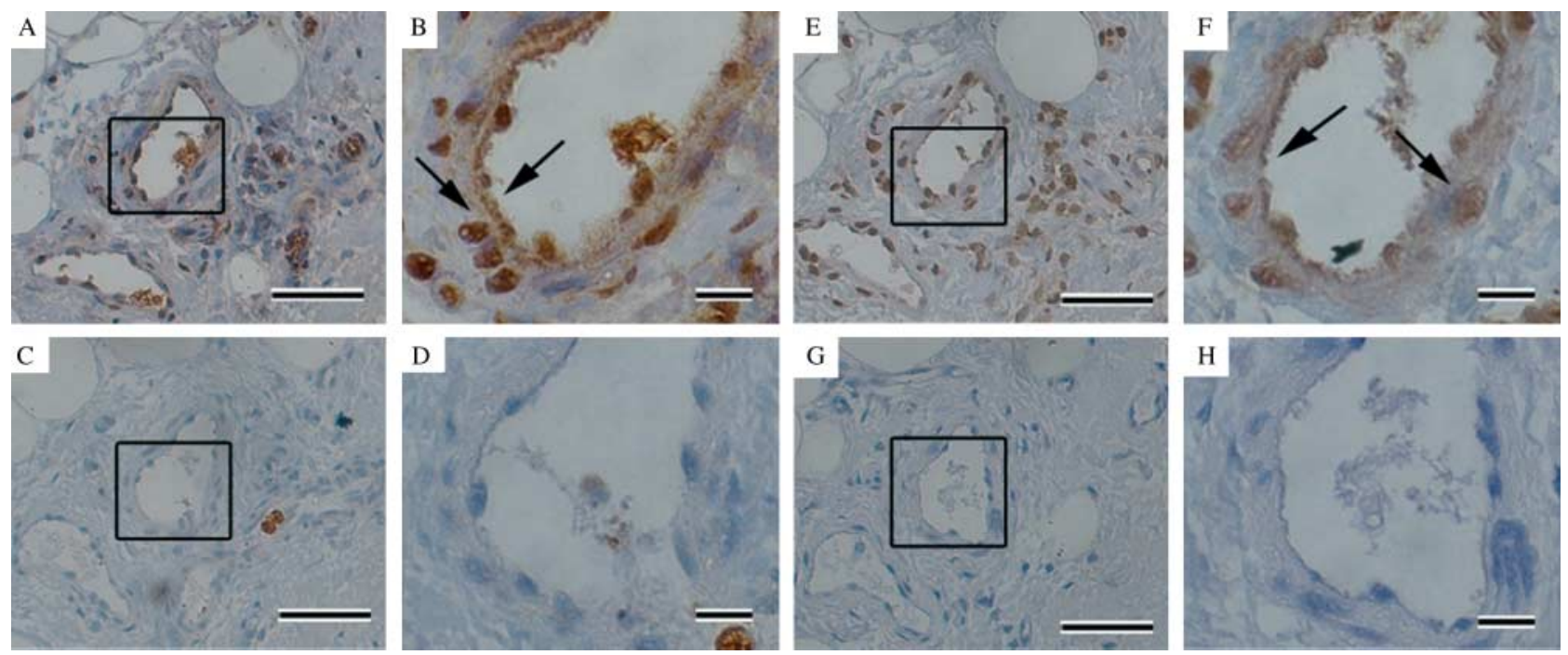

\section{Figure 4}

GLUT expression in surrounding blood vessels. (A) Strong GLUT1 immunoreactivity was observed in the endothelial cells surrounding endometriotic lesions. (B) A higher magnification showed that immunoreactivity was nuclear with some membranous staining (black arrows). (C and D) Very little GLUT3 expression was observed in endothelial cells. (E) Moderate GLUT4 immunoreactivity was observed in endothelial cells surrounding the

The absence of significant immunofluorescence in the negative control sample in the same regions confirmed the specificity of both the GLUT4 and CK19 immunofluorescence in the endometriotic epithelial cell membranes (Fig. 6H)

\section{Discussion}

GLUT proteins are the principle route for glucose entry into the cells. Very little is known, however, about the expression of these transporters in endometrial tissue and even less in endometriotic lesions. The results of this study show a significant variation in the expression of both the $S L C 2 A 3$ and SLC2A4 genes in the eutopic endometrial tissue of women with and without endometriosis. It also reports an upregulation of $S L C 2 A 3, S L C 2 A 4$ and $S L C 2 A 5$ in the ectopic tissue compared with the matching eutopic tissue. Further analysis of the GLUT1, GLUT3 and GLUT4 protein expression indicates that GLUT1 expression was present in endometriotic lesions, GLUT3 expression was restricted mainly to CD45-positive leukocytes and large nerve fiber trunks, and GLUT4 was expressed extensively in the ectopic endometrial epithelial and stromal cells with a strong expression in the membrane of the epithelial cells. The increased expression of SLC2A4 and the localization of GLUT4 to the epithelial cell membranes blood vessel. (F) A higher magnification showed a predominantly nuclear but with some membrane localization (black arrows). ( $G$ and $H$ ) Negative controls showed limited background immunoreactivity in either epithelial or stromal cells. (A, C, E and G) Scale bars represent $50 \mu \mathrm{M}$ and the areas outlined in black represent the region of higher magnification. (B, D, F and $\mathrm{H}$ ) Scale bars represent $10 \mu \mathrm{M}$.

could allow GLUT4 to provide a significant route of glucose entry into endometriotic epithelial cells.

In eutopic endometrial tissue, we found a significant variation in the gene expression of SLC2A3 and SLC2A4 between women with and without endometriosis. $S L C 2 A 3$, which was found to be significantly increased in women with endometriosis, is expressed in endometrial macrophages (von Wolff et al. 2003) and it is possible that the increase in GLUT3 gene expression in the endometrium of women with endometriosis observed in this study was due to the increased number of macrophages found in the endometrium of these women (Ota et al. 1996). By contrast, GLUT4 was significantly reduced. The variation in SLC2A4 expression between women with and without endometriosis is difficult to interpret in the context of older studies as it has not previously been examined in women with endometriosis, but in women without endometriosis, both the presence (Mioni et al. 2004, Mozzanega et al. 2004) and absence (von Wolff et al. 2003) of GLUT4 has been reported. It is also possible that these large variations and contradicting results for GLUT4 expression may be due to factors not controlled in this or other studies, such as serum insulin or glucose levels. The large difference observed, however, represents an interesting finding and a further investigation of the difference in SLC2A4 expression between women with and

Published by Bioscientifica Ltd. 

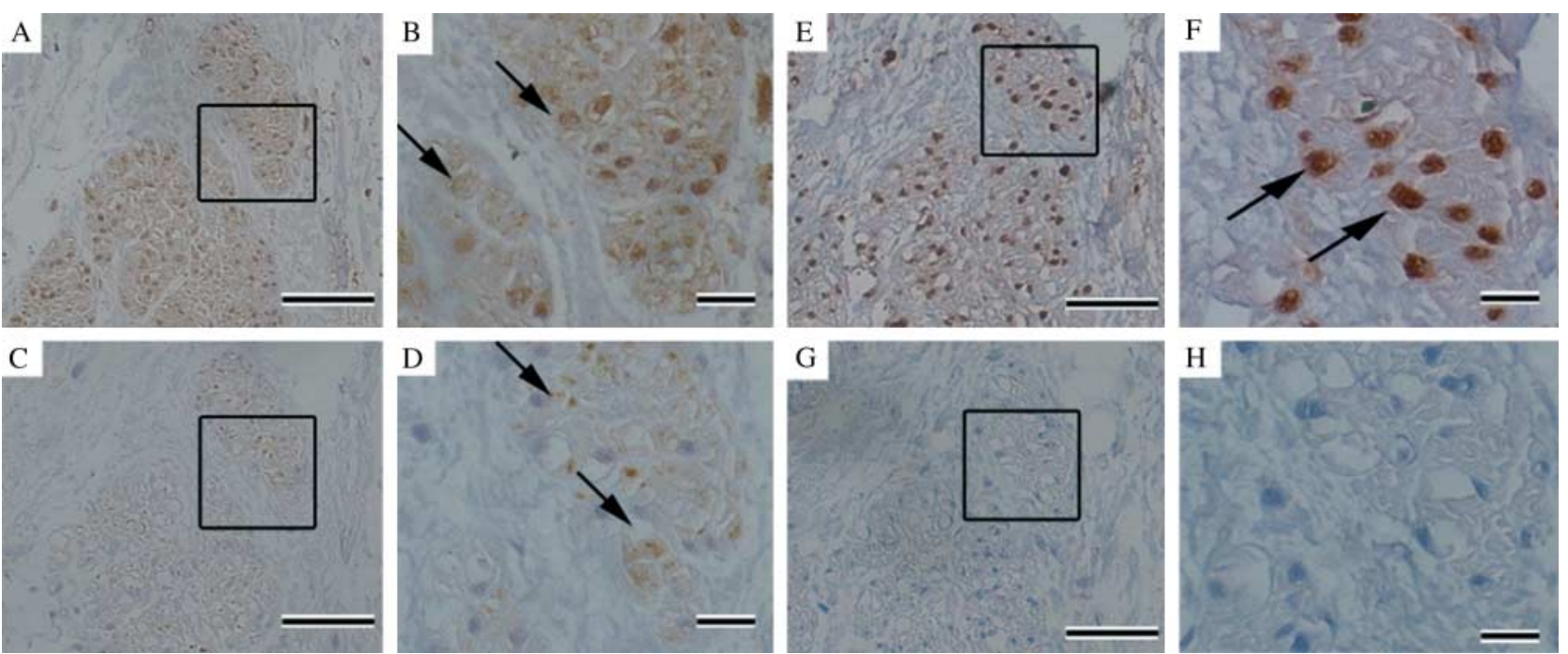

\section{Figure 5}

GLUT expression in surrounding nerves. (A) Moderate GLUT1 immunoreactivity was observed in large nerve fiber trunks surrounding endometriotic lesions. (B) A higher magnification showed that immunoreactivity was both nuclear and membranous (black arrows). (C) Weak to moderate GLUT3 immunoreactivity was observed in large nerve fiber trunks. (D) A higher magnification showed that immunoreactivity was predominantly nuclear (black arrows). (E) Moderate to strong GLUT4

without endometriosis, taking into account factors such as the insulin and glucose serum concentrations, should be performed on a larger sample size.

An analysis of gene expression in ectopic tissue found a significantly higher expression of $S L C 2 A 1$ and $S L C 2 A 8$ in the peritoneal lesions compared with the ovarian lesions. The difference supports the notion that endometriotic lesions may be a collection of related but individual diseases (Nisolle \& Donnez 1997) and that ovarian and peritoneal lesions may acquire different amounts of glucose or may even utilize different mechanisms to acquire sufficient glucose. In addition, as previous studies have shown that both ectopic lesions and the peritoneal microenvironment can be significantly affected by hormonal treatments (Nirgianakis et al. 2013), we also examined whether these had an effect on SLC2A expression. No significant variation, however, was observed in the expression of any genes after either HC treatment or GnRHa use, indicating that the effectiveness of these drugs is not involved with a modulation of the metabolic capabilities of the lesions.

In a comparison between the SLC2A gene expression in eutopic and matching ectopic tissue, we found a significant upregulation of expression in SLCA3, SLC2A4 and SLC2A5. Of these three genes, there was a large immunoreactivity was observed in the large nerve fiber trunks. (F) A higher magnification showed almost exclusive nuclear localization (black arrows). ( $G$ and $H$ ) Negative control showed limited background staining in either epithelial or stromal cells. (A, C, E and G) Scale bars represent $50 \mu \mathrm{M}$ and the areas outlined in black represent the region of higher magnification. (B, D, $\mathrm{F}$ and $\mathrm{H}$ ) Scale bars represent $10 \mu \mathrm{M}$.

expression of both $S L C 2 A 3$ and $S L C 2 A 4$ in the ectopic tissue. SLC2A5, although significantly higher in ectopic tissue, still showed a very low expression and thus was not considered for further investigation. By contrast, SLC2A1 surprisingly showed no significant change in expression event although it is significantly increased by the hypoxic environment created during malignant lesion growth (Goldman et al. 2006, Szablewski 2013). We therefore chose to further analyze the presence of GLUT1, GLUT3 and GLUT4 at the protein level in the ectopic tissue.

SLC2A1 gene expression in endometriotic lesions has not previously been reported; however, the GLUT1 protein expression in benign endometriotic lesions was found in $16-19 \%$ of ovarian endometrioma, which increased to $95 \%$ in endometriosis-associated clear cell carcinoma (Kato et al. 2012). In the ectopic tissue, we found no significant variation in the SLC2A1 gene expression compared with the matching eutopic tissue, but did find GLUT1 expression in most of the cell types examined with immunohistochemistry. These results in combination suggest that GLUT1 is present in ectopic endometriotic lesions but it is not upregulated, at least at the genetic level, by the change to benign but pathological ectopic endometriotic lesions. 

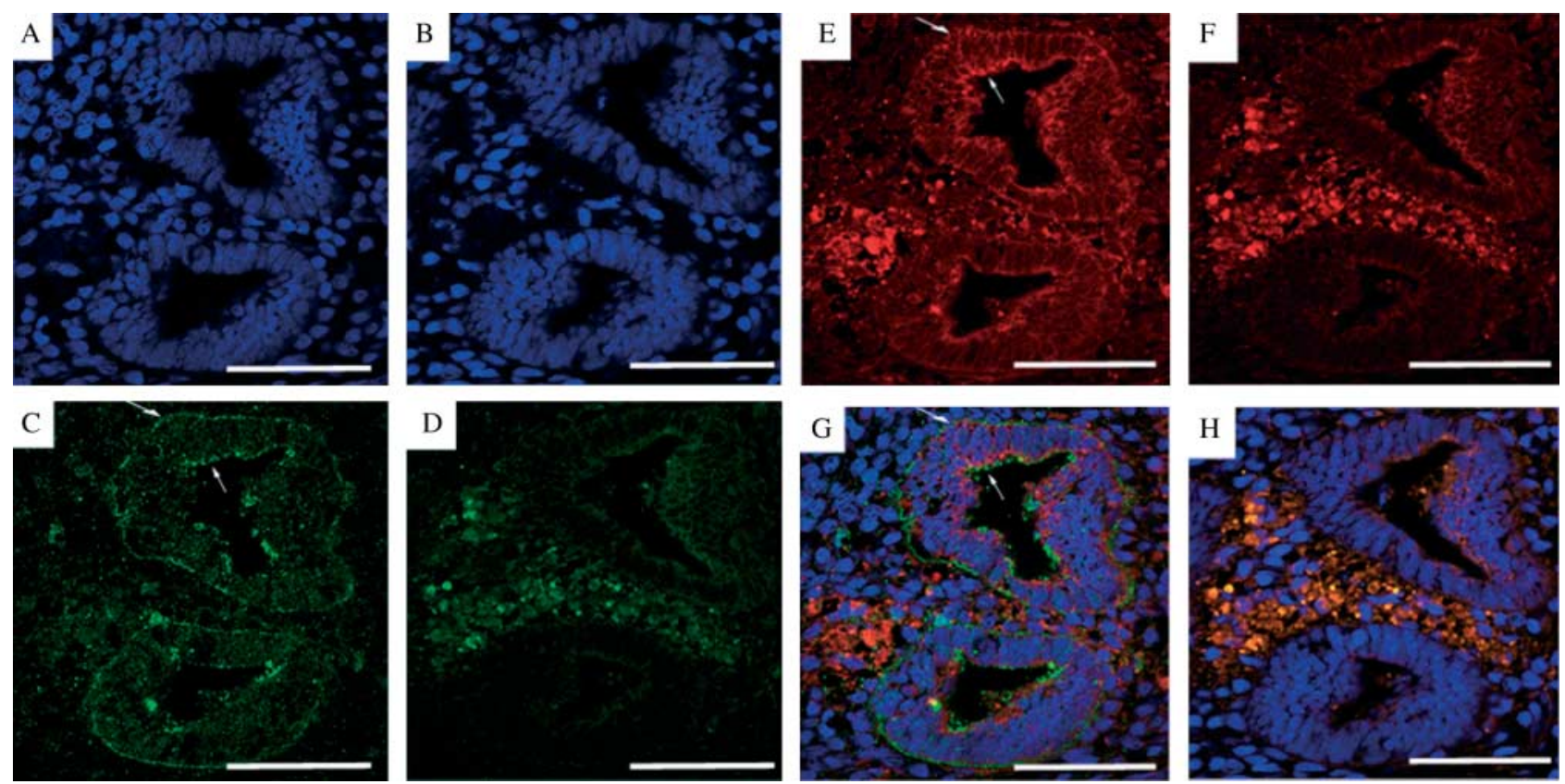

\section{Figure 6}

GLUT4 membrane localization in endometriotic epithelial cells. (A, B, E and G) Endometriotic lesions were incubated with both GLUT4 and CK19 primary antibodies and fluorescent-conjugated secondary antibodies. $(B, D, F$ and $H$ ) Negative controls were performed in the absence of primary antibodies. (A and B) Nuclei in both the positive and the negative controls were visualized with DAPI. (C) Strong GLUT4 immunofluorescence (green) above the background and autofluorescence observed in (D) the negative control was observed predominantly in apical and basal regions of the

GLUT3 is a high-affinity transporter and is usually expressed in tissue with high metabolic requirements, such as the brain (Shepherd et al. 1992). It has also been identified in normal endometrium; however, in these studies it was confined to the CD45-positive cells (von Wolff et al. 2003). The immunohistochemistry results of this study indicate that GLUT3 expression was also primarily in the CD45-positive leukocytes that infiltrate the endometriotic lesions, as well as the surrounding large nerve fiber trunks in the ectopic tissue. Increased numbers of immune cells have been detected in endometriotic lesions (Halme et al. 1983), and nerve fibers are found proximal to lesions, particularly in the peritoneal cavity and the RVS (Mechsner et al. 2007, McKinnon et al. 2012). It is hence possible that the increase in SLC2A3 expression observed in this study is derived from the increased infiltration of leukocytes and nerve fibers and therefore that GLUT3 may not contribute substantially to the glucose requirements of the ectopic tissue.

GLUT4 is an inducible, insulin-sensitive transporter predominantly expressed in skeletal muscle and glandular structures of the endometriotic lesions (white arrows). (E) CK19 immunofluorescence above the background and autofluorescence observed in (F) the negative control was also observed in the epithelial cell membranes (white arrows). (G) An overlay of the GLUT4 and CK19 immunofluorescence showed a significantly stronger signal of both GLUT4 and CK19 in the epithelial cell membranes above the background and autofluorescence observed in $(\mathrm{H})$ the negative control. Scale bars represent $50 \mu \mathrm{M}$.

adipose tissue. In response to insulin, GLUT4 translocates from intracellular compartments to the plasma membrane, resulting in a rapid increase in glucose uptake (Bryant et al. 2002). Once at the membrane, GLUT4 can increase glucose uptake into the cell by 10- to 40-fold (Shepherd \& Kahn 1999). The immunohistochemical and immunofluorescence images in this study confirm that the GLUT4 protein is found in significant concentrations in the endometriotic epithelial and stromal cells, and in particular at the epithelial plasma membrane. This expression and membrane localization indicates that GLUT4 has the potential to provide glucose to the lesions and thus may represent an important transporter for glucose entry into ectopic tissue.

In summary, the results of this study show that there is an altered expression of the $S L C 2 A$ genes between women with and without endometriosis, as well as between the eutopic endometrial tissue of women with endometriosis and their matching ectopic lesions. Moreover, we were able to show that there was a significant expression of the GLUT4 transporter at the membrane of

Published by Bioscientifica Ltd 
endometriotic epithelial cells. The inducible nature of GLUT4 and its limited cellular expression may make GLUT4 an attractive target for non-hormone-based pharmaceuticals.

\section{Supplementary data}

This is linked to the online version of the paper at http://dx.doi.org/10.1530/ JME-13-0194.

\section{Declaration of interest}

The authors declare that there is no conflict of interest that could be perceived as prejudicing the impartiality of the research reported.

\section{Funding}

This research did not receive any specific grant from any funding agency in the public, commercial or not-for-profit sector.

\section{Author contribution statement}

B $M$ conceived the experimental design, performed experiments and prepared the manuscript. C W and D B contributed to experimental procedures. N A B contributed to the collection of tissue and manuscript editing. J E contributed to tissue collection. M D M supervised the project and edited the manuscript.

\section{Acknowledgements}

The authors would like to thank the theatre staff and Anne Vaucher in the laboratory for tissue collection and the progesterone measurements.

\section{References}

Bryant NJ, Govers R \& James DE 2002 Regulated transport of the glucose transporter GLUT4. Nature Reviews. Molecular Cell Biology 3 267-277. (doi:10.1038/nrm782)

D'Hooghe TM, Debrock S, Hill JA \& Meuleman C 2003 Endometriosis and subfertility: is the relationship resolved? Seminars in Reproductive Medicine 21 243-254. (doi:10.1055/s-2003-41330)

Eskenazi B \& Warner ML 1997 Epidemiology of endometriosis. Obstetrics and Gynecology Clinics of North America 24 235-258. (doi:10.1016/ S0889-8545(05)70302-8)

Evans S, Moalem-Taylor G \& Tracey DJ 2007 Pain and endometriosis. Pain 132 (Suppl 1) S22-S25. (doi:10.1016/j.pain.2007.07.006)

Florell SR, Coffin CM, Holden JA, Zimmermann JW, Gerwels JW, Summers BK, Jones DA \& Leachman SA 2001 Preservation of RNA for functional genomic studies: a multidisciplinary tumor bank protocol. Modern Pathology 14 116-128. (doi:10.1038/modpathol.3880267)

Frolova AI \& Moley KH $2011 a$ Glucose transporters in the uterus: an analysis of tissue distribution and proposed physiological roles. Reproduction 142 211-220. (doi:10.1530/REP-11-0114)

Frolova AI \& Moley KH $2011 b$ Quantitative analysis of glucose transporter mRNAs in endometrial stromal cells reveals critical role of GLUT1 in uterine receptivity. Endocrinology 152 2123-2128. (doi:10.1210/ en.2010-1266)

Goldman NA, Katz EB, Glenn AS, Weldon RH, Jones JG, Lynch U, Fezzari MJ, Runowicz CD, Goldberg GL \& Charron MJ 2006 GLUT1

http://jme.endocrinology-journals.org DOI: 10.1530/JME-13-0194
(C) 2014 Society for Endocrinology Printed in Great Britain and GLUT8 in endometrium and endometrial adenocarcinoma. Modern Pathology 19 1429-1436. (doi:10.1038/modpathol.3800656)

Halme J, Becker S, Hammond MG, Raj MH \& Raj S 1983 Increased activation of pelvic macrophages in infertile women with mild endometriosis. American Journal of Obstetrics and Gynecology 145 333-337.

Halme J, Hammond MG, Hulka JF, Raj SG \& Talbert LM 1984 Retrograde menstruation in healthy women and in patients with endometriosis. Obstetrics and Gynecology 64 151-154.

Kato M, Yamamoto S, Takano M, Matsubara O \& Furuya K 2012 Aberrant expression of the mammalian target of rapamycin, hypoxia-inducible factor- $1 \alpha$, and glucose transporter 1 in the development of ovarian clear-cell adenocarcinoma. International Journal of Gynecological Pathology 31 254-63. (doi:10.1097/PGP. Ob013e318237d66c)

Korgun ET, Celik-Ozenci C, Seval Y, Desoye G \& Demir R 2005 Do glucose transporters have other roles in addition to placental glucose transport during early pregnancy? Histochemistry and Cell Biology 123 621-629. (doi:10.1007/s00418-005-0792-3)

McKinnon B, Bersinger NA, Wotzkow C \& Mueller MD 2012 Endometriosis-associated nerve fibers, peritoneal fluid cytokine concentrations, and pain in endometriotic lesions from different locations. Fertility and Sterility 97 373-380. (doi:10.1016/j.fertnstert.2011.11.011)

Mechsner S, Schwarz J, Thode J, Loddenkemper C, Salomon DS \& Ebert AD 2007 Growth-associated protein 43-positive sensory nerve fibers accompanied by immature vessels are located in or near peritoneal endometriotic lesions. Fertility and Sterility 88 581-587. (doi:10.1016/ j.fertnstert.2006.12.087)

Mioni R, Chiarelli S, Xamin N, Zuliani L, Granzotto M, Mozzanega B, Maffei P, Martini C, Blandamura S, Sicolo N et al. 2004 Evidence for the presence of glucose transporter 4 in the endometrium and its regulation in polycystic ovary syndrome patients. Journal of Clinical Endocrinology and Metabolism 89 4089-4096. (doi:10.1210/ jc. 2003-032028)

Mozzanega B, Mioni R, Granzotto M, Chiarelli S, Xamin N, Zuliani L, Sicolo N, Marchesoni D \& Vettor R 2004 Obesity reduces the expression of GLUT4 in the endometrium of normoinsulinemic women affected by the polycystic ovary syndrome. Annals of the New York Academy of Sciences 1034 364-374. (doi:10.1196/annals.1335.038)

Mueckler M, Caruso C, Baldwin SA, Panico M, Blench I, Morris HR, Allard WJ, Lienhard GE \& Lodish HF 1985 Sequence and structure of a human glucose transporter. Science 229 941-945. (doi:10.1126/ science.3839598)

Nirgianakis K, Bersinger NA, McKinnon B, Kostov P, Imboden S \& Mueller MD 2013 Regression of the inflammatory microenvironment of the peritoneal cavity in women with endometriosis by GnRHa treatment. European Journal of Obstetrics, Gynecology, and Reproductive Biology 170 550-554. (doi:10.1016/j.ejogrb.2013.08.010)

Nisolle M \& Donnez J 1997 Peritoneal endometriosis, ovarian endometriosis, and adenomyotic nodules of the rectovaginal septum are three different entities. Fertility and Sterility 68 585-596. (doi:10.1016/ S0015-0282(97)00191-X)

Ota H, Igarashi S, Hayakawa M, Matsui T, Tanaka H \& Tanaka T 1996 Effect of danazol on the immunocompetent cells in the eutopic endometrium in patients with endometriosis: a multicenter cooperative study. Fertility and Sterility 65 545-551.

Ruijter JM, Ramakers C, Hoogaars WM, Karlen Y, Bakker O, van den Hoff MJ \& Moorman AF 2009 Amplification efficiency: linking baseline and bias in the analysis of quantitative PCR data. Nucleic Acids Research 37 e45. (doi:10.1093/nar/gkp045)

Sampson JA 1927 Peritoneal endometriosis due to the menstrual dissemination of endometrial tissue into the peritoneal cavity. American Journal of Obstetrics and Gynecology 14 422-469.

Scheepers A, Joost HG \& Schurmann A 2004 The glucose transporter families SGLT and GLUT: molecular basis of normal and aberrant 
function. Journal of Parenteral and Enteral Nutrition 28 364-371. (doi:10. $1177 / 0148607104028005364)$

Shepherd PR \& Kahn BB 1999 Glucose transporters and insulin action implications for insulin resistance and diabetes mellitus. New England Journal of Medicine 341 248-257. (doi:10.1056/ NEJM199907223410406)

Shepherd PR, Gould GW, Colville CA, McCoid SC, Gibbs EM \& Kahn BB 1992 Distribution of GLUT3 glucose transporter protein in human tissues. Biochemical and Biophysical Research Communications 188 149-154. (doi:10.1016/0006-291X(92) 92362-2)

Szablewski L 2013 Expression of glucose transporters in cancers. Biochimica et Biophysica Acta 1835 164-169. (doi:10.1016/j.bbcan.2012)
Uldry M \& Thorens B 2004 The SLC2 family of facilitated hexose and polyol transporters. Pflügers Archiv: European Journal of Physiology 447 480-489. (doi:10.1007/s00424-003-1085-0)

Vandesompele J, De Preter K, Pattyn F, Poppe B, Van Roy N, De Paepe A \& Speleman F 2002 Accurate normalization of real-time quantitative RT-PCR data by geometric averaging of multiple internal control genes. Genome Biology 3. RESEARCH0034. (doi:10.1186/gb-2002-3-7research0034)

von Wolff M, Ursel S, Hahn U, Steldinger R \& Strowitzki T 2003 Glucose transporter proteins (GLUT) in human endometrium: expression, regulation, and function throughout the menstrual cycle and in early pregnancy. Journal of Clinical Endocrinology and Metabolism $\mathbf{8 8}$ 3885-3892. (doi:10.1210/jc.2002-021890)

Received in final form 16 December 2013

Accepted 8 January 2014

Accepted Preprint published online 10 January 2014
Published by Bioscientifica Ltd. 